\title{
Diacronie
}

Studi di Storia Contemporanea

$\mathrm{N}^{\circ} 9,1 \mid 2012$

Quando la classe operaia andava in paradiso

\section{Il consenso delle sinistre italiane all'integrazione europea (1950-1969)}

\section{Maria Serena Adesso}

\section{Q OpenEdition \\ 1 Journals}

Edizione digitale

URL: http://journals.openedition.org/diacronie/3068

DOI: $10.4000 /$ diacronie.3068

ISSN: 2038-0925

Editore

Association culturelle Diacronie

Notizia bibliografica digitale

Maria Serena Adesso, « Il consenso delle sinistre italiane all'integrazione europea (1950-1969) »,

Diacronie [Online], № 9, 1 | 2012, documento 14, online dal 29 janvier 2012, consultato il 10 décembre 2020. URL : http://journals.openedition.org/diacronie/3068 ; DOI : https://doi.org/10.4000/diacronie. 3068 


\title{
Diacronie
}

\section{4/}

\section{Il consenso delle sinistre italiane all'integrazione europea (1950-1969)}

\author{
Maria Serena ADESSO *
}

L'oggetto della mia ricerca sono le sinistre italiane e il percorso, complesso, che ha portato le formazioni partitiche del nostro paese ad accettare prima, dopo alcuni anni di netta chiusura e serrati dibattiti interni, e a partecipare attivamente, poi, al processo di integrazione europea. Per rendere più agevole questa operazione, mi propongo di studiare due partiti politici: il PCI ed il PSI.

La periodizzazione è funzionale a mostrare in maniera chiara l'iter delle due formazioni politiche nel loro graduale avvicinamento al processo di integrazione europea. Ho selezionato quattro momenti fondamentali sia della storia europea che di quella italiana: il 1950 è l'anno della nascita della CECA, il 1956-1957 è il biennio del XX congresso del PCUS, della rottura del "frontismo" nel nostro paese, della firma dei Trattati di Roma, il 1962-1963 vede il convegno dell'Istituto Gramsci sulle "Tendenze del capitalismo italiano" un vero punto di svolta per i comunisti e la formazione del primo governo italiano di centro-sinistra, il 1969 segna ingresso dei delegati delle sinistre italiane all'interno dell'emiciclo parlamentare europeo. Il percorso di adesione al progetto europeo è compiuto.

\section{Introduzione}

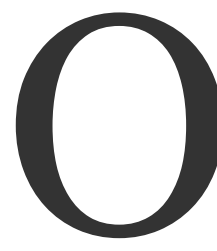

ggetto di questa ricerca sono le sinistre italiane e il percorso, complesso, che ha portato le formazioni partitiche del nostro paese ad accettare, dopo alcuni anni di netta chiusura e serrati dibattiti interni, e a partecipare attivamente, in seconda battuta, al processo di integrazione 
europea. Per rendere più agevole questa operazione, mi propongo di studiare due partiti politici, sapendo bene di tralasciare una parte dell'articolato mondo delle sinistre italiane: il $\mathrm{PCI}^{1}$ ed il PSI ${ }^{2}$. Il primo è stato il maggior partito comunista occidentale, il secondo a partire dal 1962 si è inserito nel primo governo di centro-sinistra, che segna certamente una tappa importante nella storia italiana.

La periodizzazione è funzionale a mostrare in maniera chiara l'iter delle due formazioni politiche nel loro graduale avvicinamento al processo di integrazione europea. Ho selezionato, per maggiore semplicità, quattro momenti fondamentali, quattro tappe, sia della storia europea che di quella italiana: il 1950 è l'anno della nascita della CECA; il 1956-1957 è il biennio del XX congresso del PCUS, della rottura del "frontismo" nel nostro paese, della firma dei Trattati di Roma, incipit della Comunità economica europea; il 1962 l'anno del convegno dell'Istituto Gramsci sulle "Tendenze del capitalismo italiano", un vero punto di svolta per i comunisti, e della formazione del primo governo italiano di centro-sinistra; infine il 1969 segna l’ingresso dei delegati delle sinistre italiane all'interno del Parlamento europeo. Il percorso di adesione al progetto europeo è ormai compiuto.

\footnotetext{
1 Si veda GALANTE, Severino, Il Partito comunista italiano e l'integrazione europea: il decennio del rifiuto, 1947-1957, Padova, Liviana editrice, 1988; MAGGIORANI, Mauro, L'Europa degli altri: comunisti italiani e integrazione europea 1957-1969, Roma, Carocci, 1998; SASSOON, Donald, La sinistra, l'Europa e il PCI, in GUALTIERI, Roberto (a cura di), Il PCI nell'Italia repubblicana (1943-1991), Roma, Carocci, 2001, pp. 223-249; MAGGIORANI, Mauro, FERRARI, Paolo (a cura di), L'Europa da Togliatti a Berlinguer. Testimonianze e documenti 1945-1984, Bologna, Il Mulino, 2005; PONS, Silvio, Berlinguer e la fine del comunismo, Torino, Einaudi, 2006; FERRARI, Paolo, In cammino verso Occidente. Berlinguer, il PCI e la Comunità europea negli anni '7o, Bologna, CLUEB, 2007.

2 Per il dibattito all'interno del PSI si vedano LANDOLFI, Antonio, Il socialismo italiano. Strutture, comportamenti, valori, Cosenza, Lerici, 1977; VALLAURI, Carlo, «L'interpretazione del '56 e la "via italiana". La posizione dei socialisti» in Classe, 16, 1978, pp. 94-104; GALLI, Giorgio, Storia del socialismo italiano, Roma-Bari, Laterza, 1980; EVANGELISTI, Valerio, Dalla crisi del frontismo alla vigilia del centro-sinistra, in SABBATUCCI, Giovanni (a cura di), Storia del socialismo italiano, vol. IV, Roma, Il Poligono, 1981; LANDOLFI, Antonio, Storia del PSI. Cento anni di socialismo in Italia, da Filippo Turati a Bettino Craxi, Milano, SugarCo, 1990; SABBATUCCI, Giovanni, Il riformismo impossibile. Storie del socialismo italiano, RomaBari, Laterza, 1991; DEGL'INNOCENTI, Maurizio, Storia del PSI. Dal dopoguerra ad oggi, Bari-Roma, Laterza, 1993; SCIROCCO, Giovanni, Il PSI dall'antiatlantismo alla riscoperta dell'Europa (1948-1957) in CRAVERI, Piero, QUAGLIARELLO, Gaetano (a cura di), Atlantismo ed Europeismo, Soveria Mannelli, Rubbettino, 2003; COLARIZI, Simona, Storia del Novecento italiano, Milano, Rizzoli, 2004; RAPONE, Leonardo, «I socialisti e l'Europa», in Il Ponte, LX, 23/2004, anno LX, pp. 173-194; PINTO, Carmine, Il riformismo possibile. La grande stagione delle riforme: utopie, speranze, realtà (1945-1964), Soveria Mannelli, Rubbettino, 2008; SCIROCCO, Giovanni, Politique d'abord. Il PSI, la guerra fredda e la politica internazionale (1948-1957), Milano, Unicopli, 2010.
} 


\section{1950-1953: i piani Schuman e Pleven}

Il 9 maggio 1950 il ministro degli Esteri francese, Robert Schuman, avanza la proposta di costituire un mercato unico europeo del carbone e dell'acciaio sotto il controllo di un'Alta autorità comune. Gli scopi prefissati dalla dichiarazione Schuman non sono solo meramente economici ma gettano le basi per la creazione di un'Europa unita. La messa in comune della produzione carbo-siderurgica avrebbe posto le basi per uno sviluppo economico di tutti i paesi europei che avrebbero voluto aderirvi, creando così le premesse economiche per l'auspicata federazione politica europea. L'idea di Schuman, ispirata da Jean Monnet, padre della teoria funzionalista e di certo una delle figure più importanti per il processo di integrazione europea, si pone come scopo la risoluzione di una serie di problemi stringenti: la necessità di promuovere la distensione internazionale, inserendo come elemento equilibratore tra i due blocchi un'Europa organizzata, di operare una riconciliazione franco-tedesca garantendo nello stesso tempo l'integrazione della Germania nel blocco occidentale, di creare un organismo dotato di poteri e capace di fornire un modello di sviluppo allo sforzo dell'unificazione europea.

Il 24 ottobre dello stesso anno il presidente dello Consiglio francese, René Pleven, propone un piano per la costituzione di una Comunità europea di difesa (CED) che prevede la creazione di forze armate comuni legate alle istituzioni politiche europee.

Le risposte dell'URSS alle due proposte sono drastiche e molto dure: in sostanza dietro ogni forma di integrazione europea non ci sarebbero altro che la volontà e gli interessi degli Stati Uniti e del capitalismo tedesco.

In Italia c'è un accordo sostanziale nelle dure critiche rivolte sia al Piano Schuman che alla proposta Pleven - destinata quattro anni dopo al fallimento - da parte dei socialisti e dei comunisti secondo la logica, ferrea all'inizio degli anni '50, del "frontismo". Basti leggere i commenti di Giuseppe Boffa 3 , corrispondente dell' «Unità» a Parigi, dell'economista Antonio Pesenti ${ }^{4}$ sulle pagine di «Critica economica», per quel che riguarda i comunisti, e di Pietro Nenni ${ }^{5}$ per i socialisti italiani. Le posizioni di netta chiusura resteranno tali durante tutto il processo di approvazione della proposta Schuman fino alla firma del trattato di Parigi istitutivo della CECA (Comunità europea

\footnotetext{
3 BOFFA, Giuseppe, «Gli arsenali della Ruhr e della Saar riuniti in un gigantesco cartello», in l'Unità, 10 maggio 1950.

4 PESENTI, Antonio, «Nostro paese», in Critica economica, 4, 1950, pp. 8-9.

5 NENNI, Pietro, «Fasti e nefasti del cosmopolitismo (da Trieste al Piano Schuman)», in Mondo operaio, 17 giugno 1950.
} 
del carbone e dell'acciaio) nell'aprile 1951, alla ratifica da parte del Parlamento italiano nel giugno di quell'anno e infine all'entrata in vigore della nuova istituzione europea nel luglio 1952.

Interessanti sono un paio di affermazioni di Palmiro Togliatti che mostrano, pur nell' evidente condanna del processo di integrazione europea così come è concepito dai governi, in nuce l'inizio di un'apertura - che per quel che riguarda i comunisti italiani sarà resa palese a partire solo dalla metà degli anni sessanta e resa effettiva nel 1969 nei confronti dei federalisti da un lato, e di una nuova visione dell'Europa dall'altro.

Noi non vogliamo affatto separare l'Italia dal resto dell'Europa. Vi sono nella piccola borghesia e fra gli intellettuali uomini e correnti che sognano si possa giungere presto a una unità delle nazioni europee, nella quale dovrebbero essere superati anche i confini delle singole patrie, attraverso forme di collaborazione sempre più strette. Ebbene noi non respingiamo affatto queste proposte, ma diciamo che l'Europa deve essere presa qual è. L'Europa comincia agli Urali e finisce all'Oceano Atlantico. Avviciniamoci a tutti i popoli europei, troviamo un modo di collaborare più stretto con tutti questi paesi, dalla Russia all'Inghilterra, dai paesi di nuova democrazia alla Francia. Si faccia un tentativo simile, ma non nel nome di un piccolo gruppo di satelliti dell'imperialismo degli Stati Uniti, ma non per spezzare in due il Continente e preparare la guerra, non per far risorgere il vecchio spettro del militarismo tedesco, nemico di tutti i popoli europei. Ma nell'interesse della pace, dell'uguaglianza, della fraternità, della collaborazione di tutti i popoli europei. Prendano uomini intelligenti e audaci iniziative reali e concrete in questo campo e avranno da parte nostra tutto l'appoggio ${ }^{6}$.

Il paneuropeismo di Togliatti è evidentemente molto diverso dall'europeismo federalista, traccia una prospettiva che prescinde completamente dalla dimensione istituzionale e sovranazionale, allude a un'unità europea proiettata in un futuro assai lontano.

Nell'ottobre 1952 davanti al Parlamento italiano che discute di politica estera, Togliatti compie delle dichiarazioni importanti, ostili all'europeismo governativo, ma che tendono a fare ancora un passo in avanti verso la definizione di un europeismo che non sia figlio della guerra fredda, ma che anzi tenda a superarla.

\footnotetext{
${ }^{6}$ TOGLIATTI, Palmiro, «Fermiamo la mano ai nemici della pace!», in l'Unità, 25 settembre 1951.
} 
Sul terreno economico, il vostro europeismo discende dal piano Schuman, che è un accordo di gruppi monopolistici ai danni dei paesi industrialmente più deboli come il nostro [...] e tende esso pure ad approfondire la scissione dell'Europa, rendendo permanente il blocco economico di una parte dell'Europa contro l'altra, dell'Occidente contro l'Oriente [...]. Il vostro europeismo è un ostacolo che voi ponete, seguendo i dettami dell'imperialismo americano, al fatto che i popoli d'Europa possano intendersi di nuovo, possano commerciare liberamente, possano andare liberamente verso il progresso. [...] L'Europa è quella che è. Va dagli Urali all'Atlantico. Coloro che vogliono cercare di unirla dovranno lavorare, senza dubbio, con una certa pazienza, perché oggi credo che la mente di nessun uomo di stato possa giungere a concepire quale potrà essere un'Europa unificata. [...] Mercato unico europeo e tolleranza tra i regimi socialmente ed economicamente diversi: questa è la sola forma di europeismo di oggi.7

Il fiuto politico di Togliatti lo porta a queste aperture più possibilistiche: il leader del PCI ha intuito che nel quadro internazionale qualcosa sta cambiando e che probabilmente il periodo più acuto della guerra fredda è ormai alle spalle.

Di fatti nei primi mesi del 1953 finalmente pare aprirsi, nella vita politica internazionale, una fase nuova: l'elezione di Eisenhower alla presidenza degli Stati Uniti, la morte di Stalin e l'avvento, tra numerose difficoltà, di un nuovo gruppo dirigente in URSS, la proposta di Churchill di una conferenza tra i quattro Grandi, l'armistizio di Corea, sembrano segnare la fine dello schema staliniano dell'inevitabilità della guerra tra capitalismo e comunismo e dare vigore alla politica della coesistenza pacifica ${ }^{8}$.

\section{1956-1957: firma dei Trattati di Roma}

Il XX congresso del PCUS del 1956 rappresenta un vero e proprio turning point per il Partito socialista italiano e per quello comunista. Prendendo le distanze dal modello sovietico, i socialisti, come ha scritto Nenni nel suo celebre articolo Luci ed ombre del congresso di Mosca, pubblicato dapprima sull'«Avanti» e poi su «Mondo operaio», riscoprono il nesso imprescindibile tra socialismo e democrazia9.

\footnotetext{
7 TOGLIATTI, Palmiro, «Il discorso di Togliatti alla Camera», in l'Unità, 18 ottobre 1952.

8 Si veda ROMERO, Federico, Storia della guerra fredda. L'ultimo conflitto per l'Europa, Torino, Einaudi, 2009.

9 NENNI, Pietro, «Luci e ombre del congresso di Mosca», in Mondo Operaio, IX, marzo 1956, pp. 146-154. Cfr. anche ID., Relazione al XXXII Congresso Nazionale PSI, Venezia 6-1O 
La nuova fase di coesistenza pacifica apertasi con la morte di Stalin e la fine del «comunismo di guerra»10 - così Lelio Basso ha definito gli anni in cui i comunisti si aspettavano lo scoppio di un terzo conflitto di portata mondiale - toglie argomenti alla strategia di unità a sinistra imposta dalla guerra fredda. Per i socialisti è possibile tentare una nuova "via al socialismo" e rompere il frontismo con il PCI, da un lato per aprire un dialogo con la DC e candidarsi al governo del paese, dall'altro per tenere il passo dei socialismi europei e poter rientrare nell'Internazionale socialista e, in un immediato futuro, essere parte attiva e propositiva nel processo di integrazione europea che, con il Trattato di Roma, segna un momento decisivo del proprio percorso.

Facendo un passo indietro, già nel 1953 la direzione del PSI approva nella riunione del 9 luglio la formula della «delimitazione strettamente difensiva e geograficamente limitata degli impegni militari», vale a dire, in termini più semplici, la NATO. Ciò consente un'inedita libertà d'azione: se la discriminante non è più tra occidentali e filosovietici, ma tra gli interpreti più "oltranzisti” degli impegni militari e i sostenitori della proiezione dell'alleanza atlantica sul piano della collaborazione economica e sociale, allora è possibile - per i socialisti italiani - cercare nuovi interlocutori sia sul piano nazionale che su quello internazionale. ${ }^{11}$

Considerando il 1956 l'“anno grande”, segnato dal XX congresso del PCUS, dall'invasione sovietica dell'Ungheria e dalla crisi di Suez, è nel 1957 che si consuma formalmente la rottura del frontismo, durante il congresso di Venezia del 6-10 febbraio che riassume umori, posizioni politiche, auspici e speranze maturate nel lunghissimo e denso anno precedente. Nella memoria dei socialisti italiani, sarà il congresso veneziano a fungere da spartiacque nella storia del partito e a segnare una cesura netta con il passato ${ }^{12}$.

La relazione di Nenni parte da una inequivocabile dichiarazione di fede nella democrazia: senza democrazia, senza libertà tutto si svilisce - afferma con risolutezza tutto si corrompe e diventa reazionario. A questo proposito, Nenni non compie più un distinguo tra democrazia socialista e democrazia borghese (come aveva fatto in precedenza De Martino), e polemizza con i comunisti, ai quali rimprovera l'ideologia dello Stato guida e l'accettazione di quelle pratiche che sotto il nome di dittatura del

febbraio 1957, in PARTITO SOCIALISTA ITALIANO, XXXII Congresso Nazionale, MilanoRoma, Edizioni Avanti!, 1957, p. 26.

10 Cfr. PROCACCI, Giuliano, La coesistenza pacifica. Appunti per la storia di un concetto in SESTAN, Lapo (a cura di.), La politica estera della perestrojka, Roma, Editori Riuniti, 1988, pp. 35-83; DI NOLFO, Ennio, Storia delle relazioni internazionali. 1918-1999, Roma-Bari, Laterza, 2000 .

${ }^{11}$ Cfr. DEGL'INNOCENTI, Maurizio, op. cit. pp. 186-187.

12 LANDOLFI, Antonio, op. cit. pp. 290 ss. 
proletariato altro non significano che dittatura di un partito o di un uomo ${ }^{13}$. Ribadisce che se già nel 1955 con la "politica delle cose" si era tentato un vasto accordo con gli altri partiti e con gli altri ceti sociali sui problemi concreti dei lavoratori e si era fatta una "opposizione propulsiva" al governo, ora i socialisti sono molto più aperti al dialogo con i socialdemocratici.

In politica estera Nenni rilancia il neutralismo che era stato di Costa, di Treves e Turati, cioè di "guerra alla guerra" come diceva Rosa Luxemburg, ma nella convinzione che la pace va costruita, rafforzando l'ONU, valorizzando il blocco dei paesi afro-asiatici non allineati come l'India di Nehru, ma soprattutto attraverso il ruolo autonomo dell'Europa

L'europeismo che ci interessa, e al quale siamo pronti a collaborare, è quello che tende a fare dell'Europa un elemento autonomo della politica mondiale al servizio della pace. L'Europa da noi auspicata è una Europa nella quale il peso politico dei socialisti divenga una garanzia di progresso sociale e di pace. Questa Europa è in germe nella riscossa laburista in Inghilterra, nella ripresa socialdemocratica in Germania, nel rilancio socialista in Italia, nell'evoluzione democratica dei paesi dell'Est europeo di cui la Polonia è l'esempio più recente. Ho già detto con quale spirito il nostro partito esaminerà in sede politica e sindacale le proposte per il Mercato unico europeo e per l'Euratom, senza pregiudiziali negative, ma con la cautela autorizzata dalla complessità degli interessi in causa e dalla ignoranza in cui il Parlamento e il Paese sono tenuti sugli aspetti tecnici dei problemi che involgono grosse responsabilità non tanto del Ministero degli Esteri, quanto dei ministeri dell'Industria, dell'Agricoltura e del Lavoro, di tutto il Governo e il Parlamento, dei sindacati, i quali ancora non sono stati consultati, delle stesse associazioni padronali. ${ }^{14}$

Il primo vero banco di prova della presunta "occidentalizzazione" del PSI è il voto sull'adesione dell'Italia all'Euratom e al MEC: voto favorevole per quel che riguarda l'Euratom e astensione per il MEC. La rottura con il PCI è consumata definitivamente.

Pochi giorni prima della ratifica del Parlamento italiano dei Trattati di Roma, che, firmati nel marzo 1957, sono davvero l'inizio della futura comunità europea, nel luglio 1957, sia la stampa comunista che quella socialista cominciano a darsi battaglia.

Il PCI è fermamente contrario alla ratifica del Trattato di Roma da parte del Parlamento italiano; il PSI, lo si è detto, è favorevole seppure con delle remore. Secondo

${ }^{13}$ NENNI, Pietro, Relazione al XXXII Congresso del PSI, op. cit., p. 123.

14 Ibidem, pp. 135-136. 
i comunisti italiani i due trattati sono ulteriori strumenti di divisione dell'Europa, aggravano la tensione internazionale, subordinano l'economia europea all'imperialismo statunitense, ostacolano la collaborazione con i paesi socialisti, appoggiano l'imperialismo francese nella repressione dei movimenti di liberazione dei popoli coloniali, consentono al capitale monopolistico di rafforzarsi ulteriormente, impediscono una politica di maggiori riforme strutturali, sacrificano l'agricoltura italiana e i settori industriali meno sviluppati del nostro paese con gravi conseguenze per parti consistenti della popolazione. Questa analisi allontana il PCI anche dalle posizioni della CGIL, più propensa ad una apertura nei confronti del processo di integrazione europea.

Il luglio 1957 è contrassegnato da numerosi articoli riguardanti il processo di integrazione europea e l'analisi delle prospettive economiche di tale processo, sia sull' «Unità», sia sull' «Avanti!». È agli elettori che i due partiti politici provano a spiegare, a volte anche in maniera semplicistica, le loro rispettive posizioni, non senza difficoltà visto che il processo di formazione dell'unità europea non è mai riuscito a coinvolgere $\mathrm{e}$ ad appassionare l'elettorato italiano che ha sempre sentito lontane le istituzioni sovranazionali.

Il dibattito parlamentare italiano sulla ratifica del Trattato di Roma è estremamente interessante. Riccardo Lombardi, per il PSI, e Gian Carlo Pajetta per il PCI, non si risparmiano alcun genere di polemica e sanciscono, verosimilmente, la rottura del frontismo.

\section{1962-1963: convegno dell'Istituto Gramsci sulle "Tendenze del capitalismo italiano" e formazione del primo governo di centro sinistra}

Il 10 marzo 1962 il governo Fanfani ottiene la fiducia: è il primo governo italiano che ha l'appoggio esterno del PSI: il centro-sinistra è, sebbene in una fase embrionale e convulsa, finalmente varato. Il XXXV Congresso del PSI (Roma, 25-29 ottobre 1963) pone fine alle incertezze, decidendo ufficialmente l'ingresso al governo dei socialisti. Con il primo governo Moro (5 dicembre 1963), si apre la fase organica del centrosinistra.

L'analisi riguardante la politica estera è molto interessante ed è ovviamente possibile notare l'evoluzione della posizione socialista a partire dalle aperture del 1956, ora che si trovano ad essere inseriti in una prospettiva di governo. Il ruolo italiano 
all'interno della NATO non sarà mai più messo in discussione dai socialisti italiani che considerano sempre l'Alleanza atlantica solo a scopo puramente difensivo e ne danno un'interpretazione geograficamente delimitata. La posizione socialista, riguardo la politica estera, è mutata in maniera sensibile. Dalla campagna sostenuta a fianco del PCI per anni contro la NATO, alla sua accettazione, passa la svolta che Nenni e il gruppo autonomista hanno imposto al partito, non senza scontri frontali tra le diverse anime di quest'ultimo. L'adesione alla NATO è accettata all'interno del partito obtorto collo, come sostiene anche Antonio Varsori ${ }^{15}$. Eppure la scelta a favore dell'integrazione europea diviene sempre più uno degli elementi di forza della politica estera socialista perché permette di creare un fecondo terreno d'intesa con le forze politiche moderate europee.

Per quel che riguarda la CEE, la posizione socialista è molto chiara.

I problemi della sicurezza dipendono nel nostro continente anche da ciò che sarà l'Europa, se unita o disunita, se democratica o autoritaria. Il Partito chiede un'azione energica e costante in favore dell'Europa dei popoli fondata sulla democrazia e un'azione italiana nel MEC per evitare che esso si riduca a un'area chiusa. Chiede inoltre un'azione negli organismi comunitari CEE (ai quali i socialisti intendono partecipare, operando per rimuovere preclusioni che lo hanno fino ad oggi impedito) perché diventi lo strumento delle forze della democrazia europea; chiede infine un'azione decisa dell'Italia perché sia data esecuzione ai Trattati di Roma per l'elezione di un Parlamento europeo a suffragio universale diretto. ${ }^{16}$

Il PSI ha ormai accettato in maniera netta il processo di integrazione europea e i socialisti sono pronti a farne parte (anche se entreranno per la prima volta all'interno del Parlamento europeo solo nel 1969 assieme con i comunisti) e a farsi portavoce delle esigenze delle forze politiche a cui l'emiciclo parlamentare europeo era allora precluso $^{17}$. La battaglia per l'elezione diretta dei rappresentanti al Parlamento europeo

15 VARSORI, Antonio, L'Italia e la costruzione europea negli anni del centro-sinistra: una proposta interpretativa in ROMERO, Federico, VARSORI, Antonio (a cura di), Nazione, interdipendenza, integrazione. Le relazioni internazionali dell'Italia (1917-1989), Roma, Carocci, 2005, pag. 276.

${ }_{16}$ Mozione di Autonomia socialista nel XXXV Congresso nazionale del PSI, in PARTITO SOCIALISTA ITALIANO, XXXV Congresso Nazionale, Roma 25-29 ottobre 1963, Resoconto integrale, Milano, Edizioni Avanti!, 1964, pp. 589-590.

${ }_{17}$ L'esclusione delle sinistre dal Parlamento europeo sino al 1969 si deve all'interpretazione del trattato da parte dei relatori di maggioranza che, sminuendo visibilmente il ruolo di tale istituzione, stabiliscono che esso non esercita altro che una funzione di rappresentanza dei governi dei singoli stati e che, di conseguenza, i componenti della delegazione nazionale (che in questa fase vengono ancora nominati dai governi fino alle prime elezioni dirette del 1979) vanno 
comincia a prendere le mosse dal 1963 e proseguirà per oltre dieci anni, fino alle prime elezioni del 1979.

Non è un caso che sul quotidiano socialista Luigi Vismara, ancora prima che il PSI abbia un ruolo di governo, inizia a curare un lunghissimo reportage sulle problematiche del processo di integrazione europea e sugli attori di tale processo, come se fosse importante cominciare a preparare gli iscritti, i simpatizzanti, ad una nuova e più attiva partecipazione socialista italiana al processo stesso. Il reportage si snoderà da aprile a giugno 1963 con un appuntamento settimanale sull' «Avanti!»18.

Il primo governo Moro ha inizio il 5 dicembre 1963 e resta in carica per soli sette mesi: la sua caratteristica, che costituisce anche la causa della sua caduta, è il contrasto aspro e serrato tra la DC e il PSI sulle questioni di politica economica.

Per quel che riguarda i comunisti italiani, il 1963 è un anno ricco di dibattiti interni in cui è possibile cominciare a ravvisare un leggero cambiamento di atteggiamento nei confronti del processo di integrazione europea.

Maggiorani nel suo L'Europa degli altri. Comunisti italiani ed integrazione europea (1957-1969) individua nel convegno dell'Istituto Gramsci del 1962 sulle Tendenze del capitalismo italiano ${ }^{19}$ e in una serie di articoli apparsi sull' «Unità»20, che riportano abbastanza fedelmente le mozioni approvate durante le discussioni all'interno del Comitato Centrale, un primo nodo significativo di cambiamento nella posizione di ferma intransigenza del PCI nei confronti della CEE. Giorgio Amendola figura tra i promotori e relatori del convegno ed è uno dei politici più sensibili alla questione europea. La posizione dei comunisti passa lentamente da una chiusura netta al processo di integrazione ad una posizione più possibilista che chiede che i Trattati siano rivisti e riformulati in maniera più adeguata e che ci sia una partecipazione di tutte le forze democratiche europee per un'azione comune in favore di un'Europa unita.

Attorno ad Amendola ruota negli anni Settanta il CeSPE (Centro di studi di politica economica) che con il suo «Bollettino» sarà una sede di informazione e dibattito delle questioni europee. Nel 1971 Amendola scrive un libro, dal titolo I comunisti italiani e l'Europa ${ }^{21}$, in cui, oltre a esporre la strategia comunista nei confronti della CEE, spiega il funzionamento del Parlamento europeo e degli altri organismi della comunità, per poi

scelti solo all'interno della maggioranza governativa, escludendo i partiti di opposizione. Cfr. BALLINI, Pier Luigi, GUERRIERI, Sandro, VARSORI, Antonio, Le istituzioni repubblicane dal centrismo al centro-sinistra (1953-1968), Roma, Carocci, 2006, pp. 124-141.

18 VISMARA, Luigi, in «Avanti!», 6-12-16-23 aprile, 10-15-18-26-29 maggio, 19 giugno 1963.

19 Cfr. ISTITUTO GRAMSCI, Tendenze del capitalismo italiano, Atti del convegno economico dell'Istituto Gramsci, 2 voll., Roma, Editori Riuniti, 1962.

${ }^{20}$ Cfr. DIREZIONE DEL PCI, «Per una iniziativa democratica europea e una revisione dei trattati del MEC», in l'Unità, 23 febbraio 1963.

${ }^{21}$ AMENDOLA, Giorgio, I comunisti e l'Europa, Roma, Editori Riuniti, 1971. 
passare a descrivere l'azione della Comunità stessa nei suoi vari settori di intervento. Lapalissiano è il suo contenuto pedagogico, quasi che bisognasse insegnare ai dirigenti prima ed ai militanti poi, cosa realmente fosse la Comunità Europea, che del Parlamento europeo e della PAC probabilmente poco o nulla sapevano.

\section{1969: i socialisti e i comunisti italiani fanno il loro ingresso all'interno del PE}

Il 1969 è verosimilmente un vero e proprio spartiacque nella storia del processo di integrazione europea. Da un lato fanno il loro ingresso all'interno dell'emiciclo parlamentare europeo i socialisti e i comunisti italiani, dall'altro molti storici ritengono che nel dicembre dello stesso anno, con il Congresso dell'Aja, cominci una nuovo rilancio dell’intera comunità dopo un periodo di estremo immobilismo.

Sino ad alcuni anni fa la storiografia sulla costruzione europea indicava gli anni Sessanta e Settanta come un periodo di sostanziale stasi, se non di crisi, nel processo di integrazione. A dispetto delle speranze e delle ambizioni in occasione del vertice dell'Aja del dicembre 1969, nel corso del decennio successivo, l'integrazione europea si sarebbe arenata sugli scogli rappresentati dal «serpente monetario», dalla grave crisi economica seguita alla guerra dello Yom Kippur, dalle incertezze caratterizzanti il sistema occidentale e determinate dall'apparente decadenza degli USA e dai timori suscitati per una «seconda guerra fredda». Solo la creazione dello SME nel 1978 e la prima elezione diretta del Parlamento europeo nel 1979 avrebbero gettato uno spiraglio di luce sulla sorte della CEE, aprendo la strada per le realizzazioni degli anni Ottanta.

Quanto al ruolo internazionale svolto dall'Italia in questo periodo, sarebbe stato profondamente segnato dalla crisi interna, crisi che ha posto seriamente in difficoltà le basi stesse dello Stato repubblicano: l'Italia sarebbe stata un oggetto piuttosto che un soggetto delle relazioni internazionali e la dimensione europea avrebbe rappresentato un'eccezione in tale quadro negativo ${ }^{22}$.

Scrive Maggiorani a proposito dell'ingresso dei primi deputati comunisti nel Parlamento europeo che, come argonauti reduci da un viaggio ventennale, i parlamentari del PCI approdano su un pianeta probabilmente ostile e tradizionalmente controllato dalle forze conservatrici. Un luogo sconosciuto, a volte ignorato, ma negli anni immediatamente precedenti il 1969 sempre più desiderato. Una meta cui il

${ }^{22}$ Cfr. VARSORI, Antonio, «La questione europea nella politica italiana (1969-1979)» in Studi storici, 42, 4/2001, pp. 953-971. 
movimento operaio italiano giunge al termine di una battaglia politico-parlamentare fiaccante che si risolve solo nel secondo semestre del $1968^{23}$.

I tempi sono maturi perché siano eliminati tutti gli sbarramenti e i veti nei confronti del movimento operaio e si ponga mano al rinnovo della delegazione italiana al Parlamento europeo: d'altronde, anche grazie alle insistenze comuniste, i temi comunitari cominciano a lasciare le stanze ministeriali ed essere discussi nel dibattito parlamentare, condizione, questa, che contribuisce alla "presa di contatto" del PCI con la realtà europea.

La fine dell'annosa questione della rappresentanza non ha posto fine a tutte le resistenze: al raggiunto accordo per l'ammissione dei comunisti in sede parlamentare europea $^{24}$ non segue un'intesa né per il Consiglio d'Europa ${ }^{25}$ né per l'Unione europea occidentale.

Alla fine del mese di gennaio sono comunque ufficializzati i nomi dei deputati e dei senatori comunisti a varcare finalmente la soglia del Parlamento di Strasburgo: Giorgio Amendola, Nilde Jotti, Mauro Scoccimarro, Silvio Leonardi, Francesco d'Angelosante, Giovanni Bertoli e Agide Samaritani26.

Giorgio Amendola sottolinea il traguardo storico finalmente raggiunto in una importante intervista a «l'Unità».

Noi ci apprestiamo oggi a essere presenti al Parlamento di Strasburgo essendo ben consapevoli dei limiti di fondo di questa istituzione - dovuti anche al fatto che tanta parte delle forze democratiche e socialiste dell'Europa occidentale ne è esclusa - e della crisi profonda in cui versa tutta la politica europeistica. Ci proponiamo comunque in questa sede di conseguire una più diretta conoscenza dei termini delle questioni che si pongono nella "Piccola Europa" e di utilizzare le possibilità di nuovi contatti con tutte le forze di sinistra per portare avanti in Europa la battaglia contro i monopoli, il militarismo e il revanscismo, per la pace, per il superamento dei blocchi, la cooperazione economica internazionale e profonde riforme sociali e politiche. ${ }^{27}$

\footnotetext{
23 Cfr. MAGGIORANI, Mauro, L'Europa degli altri, cit., p. 277.

${ }^{24}$ La Camera dei deputati elegge il 21 gennaio 1969 la nuova delegazione che è composta da 36 deputati e senatori così divisi: 15 esponenti della DC, 6 del PSU, 7 del PCI, 2 del PLI, 1 per PRI, PDIUM, PSIUP, SVP, MSI e Parri indipendente di sinistra.

25 Nuovamente discriminato in occasione del rinnovo delle delegazioni (25 ottobre 1972), il PCI sarà ammesso al Consiglio d'Europa con la delegazione eletta il 7 ottobre 1976.

${ }^{26}$ Samaritani, senatore ravennate, viene colto da un improvviso malore a Strasburgo il 15 marzo 1969. Al suo posto i senatori comunisti nominano Nicola Cipolla.

27 «Eletti ieri alla Camera i delegati a Strasburgo», in l’Unità, 22 gennaio 1969.
} 
Nonostante le difficoltà, la delegazione comunista, spronata da Giorgio Amendola che ne riveste il ruolo di leader indiscusso, si impegna con slancio e serietà, facendo dello studio rigoroso, della disponibilità al dialogo, ma anche della coerente difesa delle proprie idee e del proprio campo, le armi privilegiate della battaglia politica.

L'11 marzo 1969, così, per la prima volta i comunisti italiani siedono nell'emiciclo parlamentare di Strasburgo ${ }^{28}$. Un mese più tardi CGIL e CGT vengono accolte nel Comitato economico e sociale della CEE.

I comunisti italiani, diversamente dalle attese, non trovano ad accoglierli un clima di aperto contrasto con le loro posizioni né di aperta ostilità nei propri confronti. Prevalente è l'impressione che gli edifici comunitari siano abitati da un gran numero di persone capaci e intelligenti, uomini e donne che sentono «tutta l'amarezza di un lavoro che non [può] giungere a risultati positivi, concreti, per la stessa impostazione politica data alla Comunità» ${ }^{29}$.

Il piccolo gruppo comunista è molto attivo nel parlamento di Strasburgo, fa sentire le proprie opinioni, spesso discordanti con quelle della maggioranza. Ha una funzione di pungolo per ogni questione affrontata in Parlamento. Più schiacciata appare la delegazione socialista, messa in ombra dagli esponenti degli altri partiti socialisti europei. Del resto, l'approdo al Parlamento europeo non costituisce un cambiamento nelle dinamiche interne al partito socialista italiano poiché già a partire da dieci anni prima il sostegno al processo di integrazione europea è divenuto patrimonio non solo della classe dirigente ma anche dei militanti.

Con gli anni Sessanta si chiude, verosimilmente, una prima fase della costruzione dell'Europa unita: quella in cui le sinistre italiane tentano di inserirsi nella politica europea per favorire uno sviluppo più "sociale" dell'Europa che serva anche da pungolo e da sprone per la politica nazionale italiana affinché si portino avanti alcune riforme si pensi a quella del "welfare" - già realizzate in altri paesi europei. Il decennio successivo si apre con una fase di stallo del processo di integrazione europea superato solo nel 1978 con la creazione dello SME e nel 1979 con le prime elezioni dirette del Parlamento europeo.

\footnotetext{
28 «Vivo interesse per la presenza dei deputati del PCI» in l'Unità, 11 marzo 1969.

29 Intervistato da l'Unità, Amendola ha proseguito affermando «Essi avvertono acutamente la crisi. Molti ci hanno detto che, pur non essendo affatto comunisti, speravano che il nostro arrivo potesse contribuire a ravvivare una situazione ormai spenta e a suscitare nuovi interessi se non attorno all'istituzione attuale, almeno attorno ai temi dell'Europa, provocando un più vasto dibattito tra le masse». Cfr. AMENDOLA, Giorgio, «Perché i comunisti a Strasburgo?» in l'Unità, 22 marzo 1969.
} 


\section{* L'autore}

Phd in "Storia dell'Europa moderna e contemporanea" presso l'Università degli studi di Bari, si occupa di storia dell'integrazione europea e storia dei partiti politici. Collabora con l'Istituto Gramsci Puglia e con l'Associazione per la storia della Puglia e del Mezzogiorno nell'integrazione europea. Le sue ultime pubblicazioni sono «Il PCI e lo Stato d'Israele attraverso la lettura degli organi di stampa comunisti (1948-1973)» in CASILIO, Silvia, CEGNA, Annalisa, GUERRIERI, Loredana (a cura di), Paradigma lager. Vecchi e nuovi conflitti del mondo contemporaneo, Clueb, Bologna, 2011; ADESSO, Maria Serena, NEVE, Vincenzo, «Ricordando la "discesa in campo". Cenni sull'uso politico della storia nell'Italia del Cavaliere (1994-1996)» in Historia Magistra, 6/2011, pp. 37-49.

URL: < http://studistorici.com/progett/autori/\#Adesso >

\section{Per citare questo articolo:}

ADESSO, Maria Serena, «ll consenso delle sinistre italiane all'integrazione europea (1950-1969)», Diacronie. Studi di Storia Contemporanea: Quando la classe operaia andava in paradiso, 13/2/2012, URL:<http://www.studistorici.com/2012/02/13/adesso_numero_9/ >

Diacronie Studi di Storia Contemporanea $\}$ www.diacronie.it

Risorsa digitale indipendente a carattere storiografico. Uscita trimestrale. redazione.diacronie@hotmail.it

Comitato di redazione: Marco Abram - Giampaolo Amodei - Jacopo Bassi - Luca Bufarale - Alessandro Cattunar - Alice De Rensis Barbara Galimberti - Deborah Paci - Fausto Pietrancosta - Martina Sanna - Matteo Tomasoni - Luca Zuccolo

Diritti: gli articoli di Diacronie. Studi di Storia Contemporanea sono pubblicati sotto licenza Creative Commons 2.5 Possono essere riprodotti a patto di non modificarne i contenuti e di non usarli per fini commerciali. La citazione di estratti è comunque sempre autorizzata, nei limiti previsti dalla legge. 